\title{
Le prix du « retour » chez les agriculteurs « néo- ruraux »
}

Travail en couple et travail invisible des femmes

The Price of "Return" among "Neo-Rural" Farmers. Working Couple and

Women's Invisible Work

\section{Madlyne Samak}

\section{OpenEdition}

Journals

Édition électronique

URL : http://journals.openedition.org/travailemploi/7523

DOI : 10.4000/travailemploi.7523

ISSN : $1775-416 \mathrm{X}$

\section{Éditeur}

DARES - Ministère du Travail

\section{Édition imprimée}

Date de publication : 1 avril 2017

Pagination : 53-78

ISSN : 0224-4365

\section{Référence électronique}

Madlyne Samak, «Le prix du « retour » chez les agriculteurs « néo-ruraux » », Travail et Emploi [En ligne], 150 | avril-juin 2017, mis en ligne le 11 juillet 2019, consulté le 20 février 2020. URL : http:// journals.openedition.org/travailemploi/7523 ; DOI : 10.4000/travailemploi.7523 


\title{
Le prix du « retour » chez les agriculteurs « néo-ruraux »
}

\section{Travail en couple et travail invisible des femmes ${ }^{*}$}

\author{
Madlyne Samak ${ }^{* *}$
}

\begin{abstract}
L'article interroge les logiques sociales qui conduisent des agricultrices « néorurales », à savoir non issues de familles d'agriculteurs, à devenir maraîchères aux côtés de leur conjoint et à travailler dans l'ombre de celui-ci, sans statut professionnel. Il montre d'abord en quoi l'entrée en indépendance de ces femmes obéit parfois à des logiques familiales plus que professionnelles. Il expose ensuite les ressorts de leur invisibilité statutaire : au-delà des faibles ressources économiques qui contraignent ces petit·e·s exploitant·e.s et limitent leur capacité à payer les cotisations sociales, on note une méconnaissance des dispositifs et des risques encourus, mais aussi une certaine défiance à l'égard des formes instituées de protection sociale qui les conduit à privilégier la solidarité de couple et à élaborer des stratégies individuelles compensatoires.
\end{abstract}

"E agriculture, les travailleurs entretiennent entre eux des rapports d'alliance et de parenté et les rapports de travail sont en même temps des rapports entre les sexes et entre catégories d'âge » (BARTHEZ, 1982, p. 124). Cette analyse, produite par Alice BARTHEZ au début des années 1980, s'avère toujours pertinente aujourd'hui. En France, le secteur agricole demeure marqué par une emprise forte du travail familial. En 2010, $71 \%$ du travail était effectué par des actifs familiaux (chefs d'exploitation, coexploitants, conjoints d'exploitant, aides familiaux ${ }^{1}$, salariés familiaux), tandis que les salariés permanents sans lien familial avec le chef d'exploitation ou le coexploitant

\footnotetext{
* Je remercie vivement Sarah Abdelnour, Sophie Bernard et Julien Gros pour leurs commentaires et suggestions, ainsi que Sibylle Gollac pour sa lecture critique du texte lors de la journée d'étude «Genre et travail indépendant. Les divisions sexuées du non-salariat ».

** Institut de recherche interdisciplinaire en sciences sociales (Irisso), CNRS, université Paris-Dauphine ; madlynesamak@gmail.com.

1. Le statut d'aide familial est réservé aux personnes, âgées d'au moins 16 ans, ascendants, descendants, frères, sœurs ou alliés au même degré du chef d'exploitation agricole, ou de son conjoint, qui vivent sur l'exploitation et participent à sa mise en valeur sans avoir la qualité de salarié.
} 
y contribuaient à hauteur de $17 \%^{2}$ et les saisonniers à hauteur de $10 \%$ (LAISNEY, LERBOURG, 2012). Les exploitations comme le métier d'agriculteur se transmettent le plus souvent au sein de la famille, ce qui fait de l'agriculture un secteur singulier, caractérisé par la persistance d'entreprises familiales (BESSIÈRE et al., 2008).

Malgré cette stabilité de l'organisation du travail, la place et le statut des femmes dans les unités de production agricole ne sont pas restés figés, et les inégalités de genre dans ce secteur se sont réduites.

D'une part, les femmes agricultrices ont considérablement gagné en indépendance et en reconnaissance professionnelle durant les trente dernières années. Jusqu'au début des années 1980, les conjointes d'agriculteurs n'étaient pas considérées comme des travailleuses à part entière mais comme des «aides familiales » : le travail fourni dans le cadre de l'exploitation agricole était invisible et elles ne disposaient d'aucune garantie professionnelle ou sociale, si ce n'est comme ayants droit de leur mari. Leur statut professionnel était alors confondu avec leur statut matrimonial (LAGRAVE, 1988 ; CAniou, Lagrave, 1988) et leurs droits sociaux, notamment concernant la retraite, étaient inexistants ${ }^{3}$. Depuis lors, des changements législatifs ont consacré une lente, mais certaine, reconnaissance du travail des agricultrices. D'abord, la loi d'orientation agricole du 4 juillet 1980 a créé le statut de coexploitant, permettant en théorie aux femmes d'avoir les mêmes droits et responsabilités que leur mari. Et si, en pratique, les femmes ont dans un premier temps très peu accédé à ce statut, la création en 1985 des exploitations agricoles à responsabilité limitée (EARL), c'est-à-dire de sociétés civiles permettant aux époux d'être les seuls associés ${ }^{4}$, a entraîné un accroissement du nombre de femmes coexploitantes ${ }^{5}$. En 2010 en France, la part des femmes parmi les chef.fe-s d'exploitation et coexploitant-e.s était de $27 \%$, contre $8 \%$ en 1970 (LAISNEY, LERBOURG, 2012). On trouve désormais plus de femmes exploitantes ou coexploitantes (42,6\% en 2007) que de conjointes travaillant avec ou sans statut à la ferme $(34,4 \%)$ (BISAULT, 2009). Ensuite, la loi d'orientation agricole de 1999, instituant le statut de « conjoint collaborateur » (remplaçant celui de « conjoint participant aux travaux $\left.{ }^{6} »\right)$ a aussi permis une reconnaissance professionnelle du travail des femmes et une amélioration de leur protection sociale ${ }^{7}$. Ce statut leur offre la possibilité de toucher une retraite proportionnelle et ouvre des droits assurantiels en cas d'accident du travail. Certes, il n'accorde aucun pouvoir décisionnel sur l'exploitation et demeure un statut « mis à l'index » (CANIOU, LAGRAVE, 1988) - les agricultrices ont des droits dérivés,

2. Le travail saisonnier est mesuré en nombre d'unités de travail annuel (UTA) et non en nombre d'actifs.

3. Pour une analyse historique sur le temps long du cas spécifique des salariées agricoles, voir EscUDIER, 2017.

4. Contrairement aux groupements agricoles d'exploitation en commun (Gaec).

5. «Le développement des formes sociétaires, et son corollaire les postes de coexploitants, facilitent l'accès aux responsabilités des agricultrices. Des mères s'associent avec leur fils dans les groupements agricoles d'exploitation en commun ( $\mathrm{Gaec})$. Des épouses avec leur mari dans les exploitations agricoles à responsabilité limité (EARL) » (BisAult, 2009, p. 1).

6. Avant la création du statut de conjoint collaborateur, celui de conjoint participant aux travaux de l'exploitation ouvrait le droit à une retraite forfaitaire. Il avait été créé par la loi d'orientation agricole de 1980.

7. Depuis 2005 ce statut est ouvert aux personnes pacsées. 
elles sont ayants droit de leur mari et donc inactives pour le droit social - mais sa création a permis de réduire le nombre de conjointes travaillant sans statut dans les exploitations ${ }^{8}$.

D'autre part, si le travail agricole demeure un travail que l'on exerce en famille, force est de constater que depuis les années 1980, une part croissante des compagnes d'agriculteurs s'est tournée vers le marché du travail salarié. Dans l'enquête Emploi de 2000, seulement 43,4\% des conjointes d'un chef d'exploitation se déclaraient agricultrices «en activité », soit onze points de moins que dix ans plus tôt (GIRAUD, RÉMY, 2008, p. 30). Le recensement agricole de 2010 révèle quant à lui que près des trois quarts des conjointes de moins de 40 ans ne travaillent pas sur l'exploitation de leur mari agriculteur (LAISNEY, LERBOURG, 2012). En clair, le modèle de l'exploitation conjugale est aujourd'hui en net recul (DUFOUR, GIRAUD, 2012). Si la parité n'est pas de mise en agriculture (RATTIN, 2006), on ne saurait toutefois contester que les conjointes d'agriculteurs, en travaillant de plus en plus souvent à l'extérieur de l'exploitation ou en accédant au statut de coexploitante, ont gagné en indépendance et en reconnaissance professionnelle au cours des trois dernières décennies.

Derrière cette tendance lourde, il est pourtant des espaces sociaux où les conjointes d'agriculteurs continuent d'aligner leur activité professionnelle sur celle de leur conjoint, et d'agir sans visibilité professionnelle. En ce sens, l'enquête menée auprès des maraîchers biologiques des Alpes-Maritimes a donné à voir une tout autre réalité. Chez la majorité des agriculteur·rice-s rencontré.e.s (encadré 1), le travail conjugal demeure la norme et cette permanence de l'agriculture comme "métier de couple » (BARTHEZ, 1982) est encore plus flagrante chez les maraîchers « néo-ruraux », ces agriculteurs qui ne sont pas eux-mêmes issus de familles d'agriculteurs et qui créent de toutes pièces leur exploitation agricole. Ce résultat n'est guère étonnant dès lors qu'on le replace dans son contexte sectoriel - le maraîchage reste un secteur très conjugalisé par rapport à d'autres (DUFOUR, GIRAUD, 2012) -, mais il l'est en revanche davantage si l'on tient compte des caractéristiques sociales des agricultrices concernées. Car si on aurait pu s'attendre à ce que les agricultrices « néo-rurales », provenant pour la plupart des classes moyennes ou supérieures urbaines et diplômées (encadré 2), soient les actrices d'une réinvention des normes de genre en agriculture, il apparaît à l'inverse que, dans ce groupe, non seulement la quasi-totalité des fermes est exploitée en couple, mais le travail féminin est aussi rendu invisible par la non-déclaration des conjointes travaillant sur l'exploitation. Cet article se propose de présenter et d'expliquer ces résultats paradoxaux et plus précisément de saisir les logiques sociales qui conduisent ces femmes à devenir agricultrices aux côtés de leur conjoint et à travailler dans l'ombre de celui-ci, sans statut professionnel. Les inégalités de genre au travail chez les groupes de « néo-ruraux » (agriculteurs, artisans) ont été soulignées dans d'autres recherches (LÉGER, HERVIEU-LÉGER, 1979 ; BAJARD, 2014), mais peu explorées sous l'angle des mécanismes sociaux de leur production et reproduction. Ce texte vise ainsi

8. Celles-ci représentaient encore 21,3\% des actives agricoles en 2007 (BISAULT, 2009). 
à pointer la fragilité des acquis professionnels des femmes en montrant dans quelle mesure et pourquoi certains groupes sociaux d'agriculteurs demeurent imperméables à la reconnaissance du travail féminin et tendent de fait à reproduire les inégalités de genre dans leur activité professionnelle.

\section{ENCADRÉ 1}

\section{Une enquête sur les maraîcher·ère·s biologiques des Alpes-Maritimes}

Les matériaux présentés dans cet article sont issus d'une enquête consacrée à l'engagement professionnel des agriculteur-rice-s biologiques, menée dans les Alpes-Maritimes entre 2009 et 2012 dans le cadre d'une thèse de doctorat. Outre le travail d'analyse de plusieurs corpus d'archives administratives et de traitement secondaire d'une enquête statistique, l'enquête reposait principalement sur la conduite d'une soixantaine d'entretiens semi-directifs et l'observation de scènes collectives variées, telles que des sessions de formation professionnelle et d'information sur la « conversion » à l'agriculture biologique ou des temps de commercialisation (vente sur le marché, distributions effectuées au sein d'Associations pour le maintien d'une agriculture paysanne [Amap]). Les entretiens, réalisés à domicile pour la plupart, se sont souvent accompagnés d'une visite de l'exploitation et d'une participation ponctuelle aux travaux, moment au cours duquel je pouvais à la fois observer l'espace de travail et me renseigner sur les pratiques professionnelles concrètes.

Apparue au début des années 1960 au sein de quelques exploitations maraîchères du département, l'agriculture biologique connaît une première période de croissance dans les années 1990, puis une seconde période d'accroissement à la fin des années 2000, qui porte à 181 le nombre d'exploitations certifiées par le label « agriculture biologique » en 2012. En 2011, le marâichage constituait la première spécialité culturale pratiquée par les agriculteur.rice-s biologiques ( $45 \%$, contre $34,3 \%$ dans l'ensemble des exploitations du département), devant l'oléiculture, la culture fruitière et l'élevage laitier (caprin). Si les faibles effectifs d'exploitations certifiées bio rendent inexploitables les enquêtes de statistique agricole en vue de produire une caractérisation précise de l'agriculture biologique locale, précisons toutefois que les vingt-deux exploitations maraîchères visitées au cours de l'enquête occupaient des positions socio-économiques variées, allant de la micro-exploitation de moins d'un hectare, située en zone de montagne à haute altitude, au domaine de sept-huit hectares disposant d'importantes surfaces sous serre et situé en zone de plaine à proximité de communes à forte densité urbaine.

Les rapports de genre au travail n'étaient pas au cœur du questionnement de cette recherche, mais les données recueillies sur les parcours et les statuts professionnels des femmes et des hommes enquêté.e.s m'ont permis d'en saisir certaines dimensions. Aussi, si la question du travail invisible en agriculture renvoie classiquement à l'absence de reconnaissance professionnelle et à l'émiettement des tâches accomplies par les femmes (LAGRAVE, 1988), elle est traitée dans ce texte sous l'angle statutaire essentiellement. 


\section{Devenir agricultrice « néo-rurale » : entre rupture professionnelle et projet familial}

Précisons tout d'abord que le caractère familial du travail agricole se confirme sur le terrain enquêté : sur les vingt-deux unités de production maraîchères que j'ai visitées (encadré 2), quinze sont exploitées « en famille »- les sept autres le sont généralement par un.e agriculteur.rice travaillant seul.e. Dans la plupart des cas, la dimension familiale est réduite à sa composante conjugale et ne s'explique pas par l'existence de rapports intergénérationnels entre travailleur-euse-s. Plusieurs raisons expliquent cela. D'une part, pour la moitié environ des personnes enquêtées, l'installation en agriculture s'est traduite par la construction d'une nouvelle exploitation : si leurs parents les ont parfois aidés dans cette démarche, ils ne se sont jamais physiquement et durablement investis dans le procès de production. D'autre part, au moment de l'enquête, la moyenne d'âge des maraîcher.ère·s ayant repris une exploitation familiale est trop élevée $e^{9}$ pour que ceux-elles-ci aient encore affaire à leurs parents ou beaux-parents sur l'exploitation (qui sont soit d'un âge très avancé, soit décédés). Enfin, lors de mes passages sur ces exploitations familiales, je n'ai pas vu les filles et fils des agriculteur.rice-s enquêté.e-s travailler avec leurs parents : ils.elles sont engagé.e.s dans des trajectoires scolaires ou professionnelles éloignées du secteur agricole. En somme, sur le terrain étudié, c'est bien le modèle de l'exploitation conjugale qui domine. Et de fait, sur les dix-neuf ${ }^{10}$ conjoint.e.s rencontré.e.s, seules quatre (uniquement des femmes) occupent un emploi salarié en dehors de l'exploitation et ne participent pas régulièrement au travail agricole ${ }^{11}$. En dehors de ces quatre femmes, douze conjoint-e.s travaillent sur l'exploitation familiale à plein-temps et n'exercent aucune activité professionnelle à l'extérieur ; trois (un homme et deux femmes) travaillent dans un autre secteur d'activité, tout en contribuant régulièrement au travail agricole et en ayant pour projet de s'impliquer davantage sur l'exploitation et d'abandonner totalement ou partiellement leur emploi salarié.

Ce résultat n'est guère étonnant si l'on considère que les conjointes d'agriculteurs travaillent plus souvent sur les exploitations maraîchères (DuFOUR, GIRAUD, 2012) et sur les exploitations les plus polyvalentes (LAISNEY, LERBOURG, 2012), où certaines tâches supposent toujours une forte participation féminine ${ }^{12}$ (BESSIÈRE et al., 2014). Mais à l'aune du terrain, on peut aussi le comprendre comme le produit du caractère délibérément conjugal des projets de « retour à la terre » et de construction de nouvelles exploitations. Chez les «néo-ruraux-ales » en effet, le modèle de l'exploitation

\footnotetext{
9. La moyenne d'âge élevée des agriculteur·rice·s enquêtée-e·s est plus un effet de lieu que de positionnement professionnel : du fait de l'urbanisation et du fort taux de déprise agricole, la moyenne d'âge des agriculteur.rice·s maralpin·e·s s'élève en effet à 58 ans (Agreste, Enquête sur la structure des exploitations 2005).

10. Trois agriculteur·rice-s rencontré·e·s au cours de l'enquête étaient célibataires ou divorcé·e·s.

11. Comme pour les boulangères étudiées par Isabelle BERTAUX-Wiame (1982), le refus des femmes de s'investir dans l'entreprise familiale est d'autant plus probable que celles-ci avaient une position professionnelle assurée avant l'installation de leur conjoint.

12. Les tâches comme la traite ou la vente sur les marchés ont des horaires contraignants et sont difficiles à salarier.
} 


\section{ENCADRÉ 2}

\section{Les agriculteur·rice·s « néo-ruraux·ales » de l'enquête}

Sur les vingt-deux exploitations enquêtées dans cette recherche, dix appartiennent à des agriculteur·rice·s non issu·e.s de familles d'agriculteurs et devenu·e.s maraîcher·ère·s après une expérience professionnelle non agricole plus ou moins longue. Le corpus mobilisé ici est celui des entretiens menés avec ces maraîcher·ère·s « néo-ruraux·ales ».

Je reprends ici entre guillemets la catégorie « néo-ruraux » pour mettre à distance tout l'imaginaire collectif que suscite cette notion. Comme l'a montré Ivan BRUNEAU (2006), ces agriculteur-rice-s qui ne sont pas filles ou fils d'agriculteur.rice-s ne sont pas toujours aussi étranger-ère·s au milieu rural et agricole qu'on le pense. Plus particulièrement dans le cadre de cette enquête, celles et ceux qui sont appelé.e.s « néo-ruraux·ales » ont souvent une connaissance et/ou une expérience du travail agricole : certain·e·s sont apparenté·e·s à des agriculteur-rice-s (grands-parents, oncles et tantes), d'autres ont suivi des études dans un lycée agricole pour devenir jardinier-ère·s professionnel·le·s.

Dans l'espace social diversifié constitué par les exploitations de l'enquête, les agriculteur-rice-s «néo-ruraux·ales » occupent des positions moyennes ou basses, se caractérisant par des terrains de taille petite ou moyenne, situés en altitude (là où seule une production saisonnière est possible) et par conséquent plus ou moins éloignés des grands bassins de consommation.

Ces agriculteur-rice's ont pour point commun de disposer d'importantes ressources scolaires. Sur les dix-huit individus actifs sur ces « nouvelles » exploitations au moment de l'enquête, quatorze ont obtenu un diplôme de niveau baccalauréat (bac général, technique, professionnel ou brevet professionnel responsable d'exploitation agricole). Parmi eux, la moitié a poursuivi ses études à l'université (psychologie, biologie, anglais, économie) ou en classe préparatoire (tou·te·s ne sont pas diplômé·e·s). Quatre agriculteur·rice·s seulement sont titulaires d'un brevet d'études ou d'un certificat d'aptitude professionnelle : ce sont les plus âgé·e·s des «néo », et aussi les plus tardivement installé.e·s. Mais les agriculteur·rice-s « néo-ruraux·ales » ne forment pas pour autant un groupe social homogène. D'une part, si le décompte des professions paternelles permet d'identifier une prédominance d'agriculteur·rice-s issu·e-s des classes moyennes (six agriculteur·rice.s ont un père ayant occupé une profession dite intermédiaire et quatre proviennent de familles de petits indépendants), on trouve aussi quatre agriculteur·rice-s dont le père était cadre du privé ou enseignant et trois issu·e·s des classes populaires (père ouvrier ou employé, mère au foyer le plus souvent). D'autre part, pour les agriculteur·rice-s ayant fait par leur installation en agriculture l'expérience d'une reconversion professionnelle, on observe aussi une hétérogénéité des positions sociales préalables : trois enquêté·e·s occupaient des positions intermédiaires ; quatre agriculteurs étaient commerçants ou artisans (dont trois jardiniers indépendants) et un était ouvrier ; quatre agricultrices étaient employées et trois cadres ou enseignantes. Par conséquent, il est difficile de conclure à un positionnement social caractéristique des agrobiologistes « néo-ruraux·ales » (cf. annexe pour une présentation détaillée des familles d'exploitant·e·s enquêtées). 
conjugale est encore plus répandu. Sur les dix «nouvelles » exploitations visitées au cours de l'enquête, une seule est conduite par une agricultrice célibataire (tableau), et une autre par un maraîcher en couple mais travaillant seul. Dans les huit autres cas, le travail est assuré par les deux membres du couple ou est en passe de l'être. Le travail conjugal paraît donc constituer une condition nécessaire à l'installation en agriculture . Comment dès lors l'accès à l'indépendance s'inscrit-il dans les parcours professionnels de ces agriculteur.rice.s?

TABLEAU - La place du travail conjugal sur les exploitations de l'enquête

\begin{tabular}{|c|c|c|}
\hline & « Néo-ruraux•ales » & «Repreneur·euse·s» \\
\hline Agriculteur·rice·s célibataires & 1 & 2 \\
\hline Agriculteur.rice.s en couple travaillant seul.e.s & 1 & 3 \\
\hline $\begin{array}{l}\text { Agriculteur.rice.s travaillant en couple } \\
\text { (ou projetant de le faire) }\end{array}$ & 8 & 7 \\
\hline Ensemble & 10 & 12 \\
\hline
\end{tabular}

Champ : ensemble des exploitations de l'enquête.

Source : enquête SAMAK, 2014.

\section{Agriculteurs « néo-ruraux » : de la communauté rurale à la reconversion professionnelle}

Si les motifs sociaux et politiques des installations en forme de « retour à la terre » sont marqués par une certaine continuité (alternative au mode de vie urbain et au travail salarié, rejet de la société de consommation) (BRUNEAU, 2006), force est de constater qu'on ne devient pas agriculteur « néo-rural » aujourd'hui exactement de la même manière qu'on le faisait il y a vingt ans.

Dans les années 1980 et 1990, l'installation agricole « néo-rurale » ne constitue pas une reconversion professionnelle proprement dite. Elle s'inscrit généralement dans la continuité d'un passage par l'une des communautés rurales organisées à la suite des mouvements de mai $1968^{13}$. Elle est parfois également envisagée directement à l'issue d'une scolarité largement marquée par l'idéologie du « retour à la nature ». Dans notre enquête, c'est le cas par exemple de Stéphane Muller ${ }^{14}$, né en Allemagne en 1959 d'un père pasteur et d'une mère au foyer. À l'âge de 18 ans (à la fin des années 1970), après des études secondaires courtes, il est entraîné par son frère (qui est un peu plus âgé et a déjà voyagé en France) à Tournot, petite commune des Alpes-Maritimes, où il fait la connaissance de plusieurs membres de la communauté « néo-rurale » qui s'est formée dans ce village. Très vite, Stéphane et sa compagne de l'époque décident de s'installer

13. Danièle LÉGER et Bertrand HERVIEU-LÉGER (1979) ont décrit dès la fin des années 1970 ces parcours d'installation en agriculture d'anciens communautaires. Voir également PAGIS (2015).

14. Les noms et prénoms ainsi que les toponymes ont été modifiés afin de respecter l'anonymat des personnes enquêtées. 
comme agriculteur.rice's dans la région et reprennent un petit cheptel de bovins. En 1986, lorsque l'occasion d'acquérir un terrain à Tournot se présente, Stéphane, son frère et leurs deux compagnes la saisissent et font une acquisition collective. Stéphane s'oriente dès lors vers l'activité maraîchère, passe son brevet professionnel agricole, obtient les subventions d'installation, et créée une exploitation à titre individuel.

Dans les années 2000 et 2010, l'installation agricole « néo-rurale » a lieu le plus souvent au terme d'une bifurcation professionnelle. On trouve deux cas de figure.

Dans le premier, qui est aussi le plus fréquent, l'installation en agriculture constitue une voie de reconversion pour des aspirants-artisans en difficulté sur le marché de l'emploi salarié. Parce qu'elle peut aisément être pratiquée sans formation spécialisée et à condition de disposer de ressources économiques nécessaires à l'acquisition d'un terrain ${ }^{15}$, l'activité maraîchère apparaît à des jeunes qui visaient les métiers de l'artisanat comme une opportunité de réajuster leurs ambitions professionnelles et de devenir indépendant·e·s sans passer par l'étape salariée que réclament bien souvent les métiers de ce secteur. Ainsi, Julia et Laurent Faure n'avaient que d'incertaines perspectives professionnelles avant de faire acquérir un terrain d'un peu plus d'un hectare par leurs parents, cadres supérieurs dans le privé. Née à la fin des années 1970, Julia interrompt sa scolarité à l'issue de la seconde et essaie en vain de se faire recruter comme apprentie boulangère, avant de tomber enceinte à l'âge de 18 ans et de se consacrer à la prise en charge de son fils à plein-temps. Laurent forme quant à lui le projet de devenir ébéniste après son échec au baccalauréat à la fin des années 1990. Mais il comprend rapidement que seul le secteur de la menuiserie pourrait lui offrir un emploi et éventuellement une formation. Après quelques mois passés dans une entreprise de menuiserie à découvrir un univers de travail routinier et « ennuyeux » et des rapports salariaux caractérisés par leur dureté («plus on avançait et moins je voulais être salarié »), il abandonne ce premier projet. Après deux ans d'inactivité, il se fait recruter dans le bâtiment comme manœuvre puis maître d'œuvre pendant environ un an, mais «sans grande conviction ». Pour ces deux jeunes aspirants aux métiers de l'artisanat dénués de ressources scolaires, l'installation en agriculture est apparue comme une alternative professionnelle crédible et d'autant plus souhaitable qu'elle offrait un destin de travailleur-euse indépendant-e leur permettant a priori de réaliser leur idéal de « vie familiale en autarcie ».

Dans le second cas, plus minoritaire dans notre enquête, l'installation en agriculture s'inscrit dans une dynamique de retrait d'un statut professionnel stable (LANDOUR,

\footnotetext{
15. Si l'activité maraîchère semble une alternative d'autant plus probable qu'elle ne nécessite, comparativement aux autres spécialités agricoles, qu'une faible quantité de capital économique, ces bifurcations ne sont rendues possibles que par la mobilisation de ressources généralement institutionnelles et familiales. Par les mécanismes de la donation, du legs ou de la mise à disposition d'actifs fonciers, ce sont souvent les parents des agriculteur·rice-s enquêté.e-s qui rendent matériellement possible l'installation en agriculture. Dans les rares cas où aucune forme de capital familial n'est mobilisable, les ressources institutionnelles peuvent venir compléter l'épargne individuelle, qu'il s'agisse des assurances chômage perçues pendant la période pré-installatoire ou d'un capital foncier mis à disposition par les pouvoirs publics locaux.
} 
2012, 2017 dans ce numéro ; Perez, 2014), de cadre ou d'employé non précaire ou même de commerçant. Elle s'assimile plutôt à une rupture professionnelle (DENAVE, 2015), mue par une soif d'engagement dans le travail (BIDET, 2011) et/ou par le désir de vivre « autrement», en changeant de cadre et de mode de vie. Ainsi, en devenant agricultrice, Laure Castain $\left(46\right.$ ans ${ }^{16}$ ), anciennement cadre dans une société de travaux publics, a-t-elle cherché à réaliser sa « vocation » (que son père, cadre également, avait contrariée dans son enfance) tout autant qu'à rompre avec une situation professionnelle devenue « débile » car trop peu « gratifiante » et rendant impossible la conciliation entre vie professionnelle et vie familiale ${ }^{17}$ :

«J'étais tout le temps sur le terrain, tout le temps en bagnole. J'avais des secteurs semi-nationaux. Ça aussi, ça commençait à me gonfler, c'était fatigant d'être tout le temps sur la route. Je ne trouvais pas ça très gratifiant de passer huit heures par jour à bouffer du goudron. [...] C'est un métier très stressant, avec des gens qui eux-mêmes étaient dans des professions très difficiles, avec beaucoup d'aléas. [...] J'étais accro au téléphone portable, j'avais mon fils d'un côté, le maxi-cosy et le téléphone de l'autre, c'était une vie débile. »

L'expérience d'une situation de travail problématique, renvoyant pour certain·e·s à de mauvaises conditions de travail (précarité, stress ici, mais aussi plafond de verre ou encore conflits hiérarchiques) et pour d'autres au déficit de sens associé au travail (ennui, conflit éthique ${ }^{18}$ ), semble donc aujourd'hui une constante de ces parcours de reconversion : au croisement de processus biographiques pluriels et intriqués qui mettent en jeu les différentes sphères de vie, le fait de devenir agriculteur s'apparente de plus en plus à une forme d'exit (HIRSCHMAN, 1995) professionnel.

\section{Les mécanismes genrés de la convergence des aspirations professionnelles}

Si l'installation «néo-rurale » peut constituer le point d'aboutissement d'une bifurcation professionnelle (réorientation ou rupture), comment comprendre la concrétisation en couple de ce nouveau projet professionnel ? Dans le cas du couple Faure, on le voit bien, la situation professionnelle était problématique à la fois pour Julia et pour Laurent puisque tous deux ont rencontré des difficultés pour s'insérer sur le marché de l'emploi. De plus, le couple partage depuis le début un même désir d'accès à l'indépendance (elle boulangère, lui ébéniste) et de vie au plus près de la nature. D'autres couples d'agriculteurs se sont trouvés dans cette situation de commune insatisfaction ou d'incertitude professionnelle, qui s'accompagne d'aspirations à travailler

16. Par convention, l'âge des enquêté·e·s a été calculé par rapport à l'année 2010.

17. Cet argument renvoie au poids du genre, ici la contrainte sexuée que constituent les soins à apporter aux enfants, sur les parcours de désengagement professionnel, comme le rappelle Julie LANDOUR (2012) dans ses travaux sur les cadres d'un institut de sondage.

18. Les cas de reconversion professionnelle pour conflit éthique au travail ont été observés lors d'une enquête conduite dans le prolongement de ma thèse. Par exemple, Ludovic, ingénieur spécialisé dans le contrôle de la qualité de l'air, a quitté son emploi en raison d'un désaccord politique sur l'orientation prise par son entreprise. Son parcours est décrit dans SAMAK (2016). 
moins ou « autrement », d'une volonté de changer de cadre de vie ou de faire de leur quotidien une alternative écologique et sociale (Pruvost, 2013). Jean-Paul et Suzanne Fèvre (55 et 52 ans), installés depuis 1999 sur une exploitation d'un hectare, étaient tous deux plus ou moins inactifs lorsqu'ils sont devenus agriculteurs. Ce flottement professionnel s'est avéré être un facteur de convergence de leurs aspirations professionnelles d'autant plus puissant qu'il s'ajoutait au désir de Jean-Paul de travailler la terre et à un imaginaire partagé de « retour à la nature » héritée d'une socialisation politique commune ${ }^{19}$. Parce qu'ils sont fondés sur une insatisfaction professionnelle préalable des deux membres du couple et parce qu'ils reposent sur des désirs communs en termes de style de vie, ces parcours d'installation se sont concrétisés aisément, sans provoquer de discorde.

Mais l'installation en agriculture suppose aussi parfois pour les femmes enquêtées de renoncer à une situation professionnelle stable et vécue sans difficulté afin de rendre possible la conciliation entre vie familiale et projet professionnel du conjoint. Elle représente alors un investissement par défaut obéissant avant tout à des logiques familiales, avant d'être le produit d'un exit professionnel pleinement souhaité. C'est par exemple ce que montre le parcours de Chloé Mongin (33 ans), que je rencontre en 2009 à l'occasion d'une formation technique organisée par l'association professionnelle des agriculteurs biologiques. Fille d'un couple d'enseignants expatriés, Chloé grandit et accomplit ses études supérieures en économie de la santé à l'étranger. Au début des années 2000, elle est recrutée comme ingénieure de recherche dans une organisation internationale basée au Cameroun. À cette époque, elle envisage d'y faire sa vie et y achète une maison qu'elle rénove de fond en comble («j'ai fait des travaux phénoménaux!»); au bout de quelques années d'expérience professionnelle, elle s'inscrit même en thèse (dans une université parisienne, à distance) pour progresser dans sa carrière de chercheuse. Pourtant, en 2008, elle décide de rejoindre la France, et de s'installer en maraîchage sur un terrain d'un hectare appartenant à la famille de son conjoint Gautier. Lors de notre seconde entrevue en 2010, elle est encore liée à son employeur par un contrat salarié à temps partiel ${ }^{20}$ tout en continuant d'assurer la partie administrative (élaboration des dossiers de demande de prêt) et commerciale (création du site internet, constitution d'une Amap) de l'exploitation agricole (qui est une exploitation individuelle déclarée au nom de Gautier). Mais elle projette, après sa grossesse en cours, de rompre son contrat et de s'impliquer pleinement sur l'exploitation :

\footnotetext{
19. Jean-Paul et Suzanne font partie de cette cohorte d'agriculteur-rice-s biologiques né.e-s entre la fin des années 1940 et le début des années 1960, ayant en commun d'avoir vécu des expériences socialisatrices les ayant doté.e.s d'un sens critique et de dispositions à contester l'ordre social. Dans leur cas, c'est l'engagement syndical à la Confédération française démocratique du travail (CFDT) dans les années 1970, puis la participation à des projets « alternatifs » ou à vocation sociale (coopérative, centre de vacances pour orphelins, mission humanitaire au Nicaragua) qui ont été à l'origine de leur esprit de révolte et en partie constitutifs de leur aspiration à devenir agriculteur-rice et à changer radicalement de style de vie.

20. Cette situation provisoire correspond à une stratégie économique qui rend possible l'installation du couple, comme le montre l'extrait d'entretien dans le texte. De fait, le salaire des conjointes d'agriculteurs est souvent une ressource économique qui contribue au maintien des exploitations agricoles (BESSIÈRE, 2010).
} 
« On a décidé que le Cameroun, on n'allait pas y rester, mais dans un premier temps, moi, je continuais à bosser en télétravail et en déplacement ; donc j’y vais tous les trois mois, un mois, à peu près, en moyenne. Et quand je suis ici, je travaille en télétravail à temps partiel, ce qui nous permet d'avoir un salaire et un revenu qui permet justement de pallier aux [sic] retards des administrations [pour valider leur installation], de survivre à leurs conneries. Maintenant que je suis enceinte, j'ai l'intention d'arrêter après mon congé maternité et de rester ici parce que je pense qu'à cette époque-là, on aura suffisamment de recul. Parce qu'on l'attend pour novembre, pour se dire, voilà on peut arrêter, parce qu'en plus, aller en déplacement un mois avec un petit... J'ai plein de copines qui l'ont fait, je vois ce que ça... c'est très compliqué, c'est dur pour le couple, c'est dur à beaucoup de niveaux et ça ne m'intéresse pas vraiment. Donc on va voir si on peut vivre ici à deux et puis faire... Mon projet, c'est par exemple de faire une safranière, parce que ça serait quelque chose qui se prêterait au terrain qui est très... C'est très intensif mais de façon périodique, donc il y a moyen de le conjuguer avec les autres activités d'ici, que ce soit la récolte des olives, le maraîchage, c'est des activités assez complémentaires. »

Si Chloé semble plutôt enthousiaste à l'idée de devenir agricultrice et imagine déjà comment elle pourrait mener à bien une activité valorisante pour elle au sein de l'entreprise conjugale en développant une activité de culture du safran, il reste que c'est surtout par choix conjugal et familial plus que professionnel qu'elle est devenue agricultrice. D'une part, si elle a accepté de quitter la ville et la vie qu'elle aimait, c'est d'abord pour rendre possible et durable sa relation amoureuse avec Gautier, qui n'a pas souhaité s'installer en Afrique centrale en raison d'un fort attachement à son cadre de vie («j'étais pas trop branché pour rester [en Afrique]. Je suis un peu un bouseux, j'avoue. [...] Je suis un peu attaché à ces vallées. [...] Moi, je suis campagnard, la ville c'est vraiment difficile ») ${ }^{21}$. D'autre part, c'est surtout parce qu'elle est réticente à l'idée d'élever son enfant à venir dans les conditions de travail qui sont les siennes actuellement et dans une répartition des tâches d'éducation qui contreviendrait à la division classique du travail domestique que Chloé a préféré renoncer à son emploi salarié. Travailler à distance et se rendre régulièrement au Cameroun tout en élevant ses enfants n'apparaît pas à Chloé comme une perspective désirable, et ce d'autant plus qu'elle a l'intention de s'impliquer de manière plutôt innovante dans l'éducation de son enfant $^{22}$, ce qui réclame un investissement en temps conséquent. En s'installant comme agricultrice aux côtés de son compagnon, Chloé souhaite moins quitter un travail jugé peu épanouissant que se donner la possibilité d'être une «bonne mère » (GoJARD, 2010), tout en laissant l'opportunité à Gautier de s'accomplir professionnellement et personnellement, et également d'être un « bon père ».

On peut penser aussi que son départ du Cameroun a été facilité par le fait que le village dans lequel Gautier proposait de s'installer lui offre un «cadre de vie

21. On peut aussi penser que Gautier souhaitait rester proche de sa fille issue d'un premier mariage, mais il ne l'évoque toutefois pas de lui-même lorsqu'il détaille les raisons pour lesquelles il n'a pas voulu s'installer en Afrique.

22. Par exemple, Chloé envisage de parler anglais à son enfant au quotidien pour lui permettre de devenir précocement bilingue. 
exceptionnel $^{23}$ » et se situe à quelques kilomètres à peine de celui de ses propres parents, enseignants à la retraite, ce qui assure un appui supplémentaire dans la perspective de sa future vie parentale, une manière de se rapprocher des réseaux d'entraide familiale. Cette proximité familiale de même que l'ancrage local de Gautier, dont le grand-père et l'arrière-grand-père étaient des ébénistes « reconnus » de leur village, ont aussi pu jouer comme capital d'autochtonie (RETIÈRE, 2003) permettant l'accès à une clientèle et à une meilleure position sociale ${ }^{24}$. Ces éléments ont probablement pesé dans la décision de Chloé de devenir agricultrice, décision qui l'engageait objectivement dans une trajectoire de déclassement socio-économique et qui la conduisait à renoncer à la carrière professionnelle qu'elle avait entamée.

C'est le même type de logique, entre construction d'un avenir social (BERTAUXWiAme, 1982) et prise en charge familiale, qui a conduit Samia Verdier (38 ans) à s'investir aux côtés de son conjoint dans le projet de création d'une exploitation agricole. Fille d'un couple d'immigrés de classes populaires (son père était ouvrier et sa mère au foyer), Samia grandit dans une ville industrielle de l'est de la France. À la fin des années 1980, après l'obtention de son baccalauréat et deux ans d'études supérieures qu'elle ne valide pas à la faculté de sciences économiques, elle rencontre Florent, fils d'un couple d'employés (père comptable et mère secrétaire), alors inscrit en formation professionnelle, et se marie avec lui. Peu investis dans leurs études et voulant vivre à la montagne, Samia et Florent partent s'installer en Savoie et vivent d'abord d'emplois précaires et saisonniers. Après quelques années, Samia obtient un contrat à durée indéterminée (CDI) dans la grande distribution où elle devient cheffe de rayon, tandis que Florent cumule les emplois précaires et se consacre à la rénovation de la maison que le couple a achetée. Lassé de ne pas trouver d'emploi stable et épanouissant, et aspirant à l'indépendance professionnelle, Florent décide au début des années 2000 de s'orienter vers l'agriculture : l'achat d'un terrain de deux hectares, sur lequel ils font construire un logement en bois, se fait grâce à la revente de leur maison et à la contraction d'un prêt immobilier.

Ma première rencontre avec Samia a lieu en 2009, et lors de cet entretien, je comprends que, bien que ne se qualifiant pas elle-même de «maraîchère », elle joue un rôle central sur l'exploitation :

«-Comment vous vous répartissez le travail entre vous?

Samia : - Au départ on savait pas trop, mais au fur et à mesure, il s'est avéré que... c'est lui le maraîcher. [...] Donc il prépare le terrain, parce que c'est lui avec les

23. Comme chez les artisans d'art qui s'installent en milieu rural, le cadre de vie peut constituer une rétribution sociale importante du travail (BAJARD, PERRENOUd, 2013).

24. Sybille GoLLAC (2013) éclaire l'importance du capital d'autochtonie dans les stratégies d'ascension sociale des classes populaires, qui se construisent non pas sur l'accumulation de capital scolaire mais plutôt sur la promotion interne ou la mise à son compte, stratégies qui mettent davantage en jeu un capital social et un prestige familial localisés. Bien que Gautier et Chloé ne soient pas issus des classes populaires (les parents de Chloé étaient enseignants et ceux de Gautier agents de catégorie B dans la fonction publique), les ressorts de leur installation semblent proches de ceux mis en évidence par Sybille Gollac. 
machines, c'est lui qui va planter, c'est lui qui va irriguer, et c'est moi qui vais désherber. Voilà. Ça, plus les marchés. Après, le ramassage, on se le fait à deux. Et le marché, c'est moi parce qu'il est pas du tout... autant il a été disert là, autant, c'est pas quelqu'un qui aime être sur les marchés.

- En ce qui concerne le travail dans la maison?

S. : - Oh ça, c'est moi [rires] ! Voyons ! C'est franchement désespérant. Parce que c'est pas considéré comme un travail. Aller faire du bois pour faire du chauffage, c'est du boulot mais alors nettoyer, ranger, laver, c'est pas du travail. »

L'investissement de Samia sur l'exploitation agricole de son mari ne se limite pas à la commercialisation des produits sur les marchés, tâche dont elle semble retirer beaucoup de satisfaction, elle participe aussi à la récolte et effectue l'essentiel du désherbage des cultures, qui représente une part importante du travail dans les champs, et se charge de l'ensemble des tâches administratives concernant la gestion de l'exploitation. Pourtant, Samia n'était pas certaine de travailler avec son époux lorsque celui-ci a décidé de s'installer en maraîchage. Elle gardait en tête la possibilité de trouver un emploi salarié. Si tous deux aspiraient à vivre à la montagne, l'agriculture n'était pas un objectif pour elle :

«Florent :- Au départ, quand on s'est installés, Samia savait pas trop, hein... Elle savait pas si elle avait envie de faire ça. On savait pas si on s'en sortirait, etc. Donc on gardait l'option de dire : peut-être que tu vas trouver du travail. Et pendant deuxtrois ans, on s'est pas décidés.

Samia : - Non, on s'est pas décidés mais on était le nez dans le guidon, donc...

F : - Et du coup, tu t'es vraiment jamais dit : "Je veux faire ça." Bon, elle continue à faire ça, mais finalement, on n'en a jamais vraiment reparlé.

$\mathrm{S}:-$ Ben ouais, parce qu'au fur et à mesure, quand on se retrouve à vendre, à voir que les gens viennent, reviennent, on se dit bon ben, on fait quelque chose qui a l'air de plaire, ça marche, c'est quand même con de lâcher un truc qui marche pour aller chercher hypothétiquement quelque chose de plus... c'est valorisant en plus. Voilà, c'est vrai que du coup... j'aime quand même un peu la terre parce que sinon, j'y serais pas restée hein, faut pas rêver. Lâcher un truc qui marche, c'est bête.

$\mathrm{F}:-$ Je lui avais laissé en tout cas cette option-là. Au départ, elle était pas très, très branchée quand même.

S : - Ah non, c'était pas du tout mon truc. »

Un peu plus loin dans l'entretien :

« S : Donc on est arrivés avec trois gosses, un nouveau-né, et attaquer ça [l'exploitation] en même temps. Pour moi, ça a été pénible, [...] parce que moi, ça me restait en travers de la gorge d'être là avec trois enfants, et en fait, ça nous a pris tellement de temps que... on n'a pas fait l'impasse complète sur les enfants, mais bon, moi, en tant que maman, je me disais bon, j' offre pas le temps que je devrais offrir à mes gosses. »

Samia a été comme absorbée par le projet professionnel de son mari. Face à l'ampleur des travaux à réaliser pour construire une maison puis une exploitation 
agricole, et alors qu'elle devait en même temps assurer l'éducation de trois jeunes enfants, elle n'a finalement jamais eu le temps ni l'énergie de se demander si elle voulait devenir agricultrice, ni de mettre en balance ce destin avec celui d'employée salariée. Pourtant, sa situation professionnelle et familiale s'était significativement améliorée lorsqu'elle vivait en Savoie : elle avait obtenu un poste stable à responsabilité et un salaire « à peu près correct », après plusieurs années d'emplois précaires et subalternes, et c'est son mari qui, tout en rénovant leur maison, s'occupait de leur deuxième enfant alors en bas âge. Mais quand bien même elle n'aspirait pas particulièrement à devenir agricultrice ( «c'était pas du tout mon truc »), elle a mis entre parenthèses sa propre vie professionnelle pour rendre possible le projet de son mari et, ce faisant, a subi une forte réassignation de genre au travail domestique. De fait, elle s'est progressivement attachée au travail de la terre, à la reconnaissance qu'elle en tire sur les marchés et elle trouverait aujourd'hui « bête » d'abandonner l'entreprise familiale. Mais c'est au final bien pour permettre à son conjoint d'accomplir son projet professionnel et son rêve d'indépendance que Samia est devenue agricultrice : l'inscription de cette activité dans sa propre trajectoire professionnelle s'est opérée au détour de contingences familiales (aider son mari et élever ses enfants) et non en fonction d'aspirations professionnelles ${ }^{25}$.

Carole Martin (30 ans), qui se dit pourtant « passionnée d'orchidées », a d'emblée quitté son emploi salarié de gestionnaire comptable chez son cousin fleuriste pour aider son conjoint Christophe Tissot, en cours d'installation sur une exploitation de trois hectares en zone de plaine, au moment où elle est tombée enceinte en 2012. Si la maternité semble favoriser l'investissement des femmes sur l'exploitation de leur conjoint, c'est parce que l'activité agricole leur semble faciliter la conciliation entre vie professionnelle et prise en charge des enfants en bas âge, quand bien même l'installation suscite généralement un éloignement des réseaux d'entraide familiale ${ }^{26}$. Et on peut penser que les agricultrices «néo-rurales » sont, de ce point de vue, moins susceptibles de refuser de s'impliquer sur l'exploitation agricole de leur conjoint que les filles/belles-filles d'agricultrices, car elles sont moins au fait des sacrifices et des dépendances que cette décision implique. Comme l'a montré Céline Bessière, si les compagnes des jeunes agriculteurs valorisent autant le fait de travailler en dehors de l'exploitation, c'est parce qu'elles ne veulent pas « subir le même sort que leurs bellesmères qui ont "trimé toute leur vie" » et qu'elles voient dans le travail salarié extérieur à l'exploitation l'instrument de leur émancipation (BESSIÈRE, 2010, p. 162). À l'inverse, les agricultrices «néo-rurales » n'ont pas de mère ou de belle-mère agricultrice pour contre-modèle, et ressentent probablement moins la nécessité de s'émanciper d'une condition qu'elles ne peuvent qu'imaginer.

25. Ces résultats rejoignent ceux de Marie GILlET et de Dominique JACQUES-Jouvenot (2004) sur les agricultrices non-héritières de patrimoine agricole : pour ces femmes originaires d'autres milieux sociaux, l'adoption du métier se fait par choix conjugal plus que professionnel.

26. Le cas de Chloé fait en cela exception puisque Samia et Carole, mais aussi Laure et Julia, se sont installées à longue distance de leur famille et de celle de leur conjoint. 
Il est enfin frappant de constater que les installations, lorsqu'elles sont menées par des femmes à la suite d'une reconversion professionnelle n'entraînent pas automatiquement une bifurcation des deux membres du couple. Si Jean-Pierre Revel a accepté de suivre sa compagne autrefois coiffeuse en région parisienne dans son projet d'installation en maraîchage bio, c'est que lui-même aspirait à prendre sa retraite et à vendre son commerce, et qu'il a vu dans ce projet l'opportunité de changer de cadre de vie. Mais pour Laure Castain, l'installation en agriculture n'a pas été sans complication conjugale : cadre supérieur dans une société de travaux publics, son conjoint n'a pas souhaité renoncer à sa carrière et la suivre dans son projet, malgré la présence d'un enfant en bas âge dans la cellule familiale. Le couple n'a donc pas survécu aux difficultés posées par l'installation agricole : Laure a fini par divorcer et obtenir la garde de son fils. Ainsi, à l'aune du terrain enquêté, on observe que la « cause commune » (GollaC, 2003) que constituent la prise en charge des enfants et la construction d'un avenir social, professionnel et familial, fonctionne bien comme facteur de convergence des aspirations professionnelles des couples d'agriculteur-rice-s «néo-ruraux·ales », mais surtout dans un sens : celui qui conduit les femmes salariées à quitter leur emploi et à s'investir dans le projet agricole de leur conjoint.

\section{Vers une résurgence du travail invisible des femmes?}

En matière de statut professionnel, la situation des agricultrices «néo-rurales » creuse parfois davantage encore l'écart entre femmes et hommes observé dans les exploitations plus anciennes.

L'écart existe déjà dans les exploitations des agriculteurs « repreneurs » visitées au cours de l'enquête, puisque les agricultrices ne disposent que rarement d'un statut professionnel équivalent à celui de leur mari. À l'exception d'une enquêtée que son père âgé a poussée à reprendre l'exploitation seule à l'âge de 19 ans et qui est aujourd'hui coexploitante avec son mari de l'EARL familiale, c'est le statut de conjointe collaboratrice qui est le plus prisé par les couples d'exploitants. Bien qu'elles soient parfois le vecteur par lequel ont lieu la reprise de l'exploitation et la transmission du métier (dans le cas des reprises par les gendres notamment), les agricultrices ne remettent pas en cause ce statut subalterne, tant il leur parait s'inscrire dans un ordre de genre immuable et légitime ${ }^{27}$.

Mais chez les « néo-ruraux·ales » qui travaillent en couple, les inégalités de statut entre femmes et hommes sont encore plus marquées. Si l'on exclut les situations où l'un des conjoints n'est pas déclaré comme agriculteur à titre principal car il bénéficie d'un autre statut professionnel plus avantageux (salarié, indépendant ou retraité), on

27. Cela n'est guère étonnant dans la mesure où, comme le suggère Philippe CARDON (2004, p. 124), « les femmes reproduisent les conditions mêmes de leur éviction de l'héritage du métier car elles reconnaissent la légitimité, de manière inconsciente, de la transmission en lignée masculine ». 
observe que cinq des six agricultrices enquêtées ne disposent tout bonnement d'aucun statut professionnel et ne sont même pas déclarées comme travaillant sur l'exploitation agricole.

\section{L'absence de statut comme contrainte et comme ressource}

Comment comprendre la position singulière de ces femmes, alors même qu'elles ont pour quatre d'entre elles occupé des positions professionnelles stables avant de devenir agricultrice ? D'une part, si le statut de coexploitante, de conjointe collaboratrice ou de salariée n'est jamais envisagé par ces couples d'agriculteurs, c'est d'abord en raison des contraintes économiques qui pèsent sur eux. Les cotisations sociales induites par la coexploitation représentent un coût que ces petit.e.s agriculteur-rice.s estiment ne pas pouvoir supporter, compte tenu des chiffres d'affaires modestes dégagés de leur activitée ${ }^{28}$. S'impose alors presque toujours ${ }^{29}$ une primauté masculine impensée, qui fait de l'homme le chef d'exploitation et de la femme la travailleuse invisible.

D'autre part, dans les entretiens, les femmes concernées par cette invisibilité professionnelle justifient leur situation tant par l'imprudence dont elles ont fait preuve au moment de leur installation - qui fait d'ailleurs parfois l'objet de regrets - que par une certaine défiance vis-à-vis de la protection sociale assurée par l'État. Brigitte Serres (62 ans) est une ancienne « soixante-huitarde » arrivée en agriculture par le biais des communautés rurales. Issue d'une famille de commerçants et titulaire d'une licence de psychologie, elle entame d'abord une courte carrière d'enseignante aux Antilles, avant de revenir en métropole quelques mois avant mai 1968, à la suite de l'échec de son mariage. Avec un groupe d'amis, elle participe alors à la création d'une communauté rurale dans le village de Tournot, où elle passe plusieurs années, pratiquant une agriculture vivrière et élevant ses enfants. Au milieu des années 1980, une fois la communauté dissoute, Brigitte et son conjoint Gérard décident de faire du maraîchage une activité professionnelle : ils cultivent des parcelles qu'ils louent en fermage, et créent une exploitation individuelle déclarée au nom de Gérard. Au bout de quelques années, l'occasion de faire l'acquisition d'un terrain de trois hectares se présente : le couple s'endette de 800000 francs, ce qui le contraint à augmenter considérablement sa production. Brigitte occupe un rôle central : elle assure l'essentiel

28. Sur ces exploitations, le travail invisible ne concerne d'ailleurs pas que les conjointes : le recours aux « coups de main », expression indigène qui renvoie aussi bien à des travaux non rémunérés effectués par des proches qu'à des travaux rémunérés non déclarés exécutés par des anonymes (SAMAK, 2016), est aussi fréquent.

29. À l'exception du couple Faure, dans lequel Julia est cheffe d'exploitation et Laurent n'est pas déclaré. On pourrait penser que la différenciation des statuts professionnels des conjointes de «néo-ruraux » s'opère en fonction de leur niveau de diplôme et de leur statut professionnel préalable à leur entrée en agriculture. Mais on dispose de trop peu de données pour vérifier ici cette hypothèse, dans la mesure où presque toutes ces conjointes ont des niveaux de diplôme analogues (baccalauréat et plus) et ont exercé des métiers à la position relativement équivalente dans la hiérarchie sociale (coiffeuse, employée qualifiée dans un supermarché, une coopérative, une imprimerie). De plus, le cas de Julia Faure est un contre-exemple car bien que peu diplômée et n'ayant jamais exercé d'autre métier que celui de maraîchère, elle est la seule à être déclarée dans son couple. 
de la commercialisation (quatre marchés par semaine) et de la transformation des fruits en confitures tout en s'investissant pendant le temps qui lui reste de disponible dans le travail proprement agricole (semis, désherbage, récolte). Son rôle sur l'exploitation se renforce lorsque, à la fin des années 1990, Gérard tombe malade et se voit contraint de réduire son temps de travail à un mi-temps. Si le couple peut compter sur les « coups de main » de nombreux amis et membres de la famille, il reste que Brigitte est, en raison des dettes contractées, contrainte de redoubler son activité. N'ayant jamais été déclarée comme agricultrice, et si elle semble aujourd'hui regretter en partie son insouciance, c'est non sans ironie qu'elle décrit le peu de crédit qu'elle accorde à la protection sociale du monde paysan :

« Moi je suis rien dans cette histoire, je suis pas déclarée. [...] Bon, je me rends compte que c'était pas forcément le meilleur choix. Mais bon, quand on s'est installés, y avait pas tellement... dans le monde agricole, il fallait se marier quoi. Et comme moi j'avais été mariée et que j'avais eu une mauvaise expérience, une première fois, je m'étais juré que je me remarierais plus. Voilà, c'était un peu con mais c'est comme ça, j'ai tenu, je me suis pas mariée [rires]. Ce qui fait que du coup, j'ai pas de statut. Et après... si, je suis couverte socialement. Mais c'est tout, $\mathrm{j}$ ' ai pas de retraite. Et quand je me suis rendu compte que... je me suis dit : "Bon maintenant, je vais peut-être régulariser cette histoire parce que maintenant, ça a un peu changé." Et quand je me suis rendu compte de ce que ça représentait la retraite d'un paysan, je me suis dit que finalement le minimum retraite, tu sais ce truc, c'est pareil, donc ça vaut même pas la peine [rires]. »

Par ces propos, Brigitte souligne la corrélation posée par la loi française entre statut professionnel et statut matrimonial. Si elle n'a jamais eu de statut, c'est parce qu'elle ne voulait pas se marier. L'échec de son premier mariage, mais aussi ses expériences passées de vie en communauté, qui écartaient le modèle conjugal traditionnel et considéraient le mariage comme une «institution bourgeoise », l'ont convaincue de vivre en union libre avec son conjoint Gérard. Or ce n'est que depuis 2005 que les couples non mariés (unis civilement ou simplement concubins) peuvent revendiquer le statut de conjoint.e collaborateur.rice. C'est pour cette raison qu'elle ne s'est jamais vraiment posé la question de son statut professionnel. Par ailleurs, son discours traduit le peu de confiance qu'elle accorde au système socialisé de protection sociale : en s'apercevant de la faiblesse des pensions de retraite des agriculteurs, Brigitte a jugé qu'il valait mieux ne pas cotiser et préféré construire un gîte à la place, pour s'assurer un revenu d'appoint. Cette posture de défiance vis-à-vis de l'État est courante chez les petits agriculteurs et travailleurs indépendants, qui préfèrent s'appuyer sur des solidarités de substitution plutôt que de s'en remettre aux dispositifs publics pour maintenir leur entreprise ${ }^{30}$. Or si Brigitte est diplômée du supérieur et a grandi en

30. Comme le souligne Florence WEBER (2008), le travail au noir se pratique principalement dans les secteurs économiques où l'on trouve une forte proportion de (très) petites entreprises, marquées par une saisonnalité de l'activité, et où la rentabilité des activités économiques n'est jamais acquise (restauration, bâtiments et travaux publics, agriculture). De plus, dans l'artisanat, le commerce ou l'agriculture, la frontière entre économie domestique invisible, non 
ville, elle est aussi fille de commerçants et petite-fille d'agriculteurs. Et on peut penser que l'expérience communautaire qu'elle a vécue dans les années 1970 - qui reposait sur la mise en commun des revenus monétaires (issus du travail salarié) des uns et des productions domestiques (agriculture vivrière) des autres - et ses prolongements - l'installation dans des activités artisanales ou agricoles des différents membres de la communauté - ont aussi contribué à forger sa croyance dans les solidarités interpersonnelles et la « débrouille», au détriment des modalités formelles de travail et de socialisation des ressources. De la même manière qu'elle continue aujourd'hui d'entretenir des relations de troc et d'entraide avec les membres de son entourage ( « néo-ruraux » ou «paysans du coin »), de recourir à des formes non marchandes de transaction (en bénéficiant ou en fournissant à son tour de nombreux « coups de main ») (WEBER, 2000 ; 2009) et de protection « rapprochée » (CASTEL, 1995), elle est aujourd'hui persuadée qu' elle pourra compter sur le maintien de son couple au moment de la retraite, et donc sur l'exploitation commune d'un gîte, censé leur procurer des revenus. L'échec de son premier mariage et les ruptures dans sa relation conjugale avec Gérard n'ont visiblement pas entamé sa vision idéalisée de la solidarité de couple.

C'est le même discours que l'on entend chez les Verdier où, là aussi, la nondéclaration de Samia est de mise.

«-Quand vous vous êtes installés, vous vous êtes mis directement tous les deux à titre principal?

Samia : - Je n'existe pas. Moi, j'ai pas de statut.

- C'est pour quelle raison?

S. : - Ben au départ, je voulais pas me mettre agricultrice à titre principal parce qu'on ne voulait pas doubler les charges. Ah oui, parce que si on double, on n'y arrive plus. Florent : - Le problème de la MSA [Mutualité sociale agricole], c'est qu'on paye, et quand on voit les prestations, on tombe. Que ce soit les accidents, si on a un accident de travail, mais c'est à tomber.

S : - Lui il paye quoi, 3500 euros par an ? 3500 euros de charge, si on le multiplie par deux, ben il faut rentrer plus de 3500 en production en plus. C'est pff...

F : - Alors on s'est dit, ce qu'on va faire pour la retraite de toute façon, c'est qu'on va se faire un gîte. Donc là, c'est en projet. Plutôt que de payer... Bon déjà, la spéculation, on est contre, et c'était avant la crise déjà. Que ce soit je ne sais quoi, leurs produits machin, leurs placements. Donc déjà tout ce qu'on a là, on l'investit sur le terrain. Et en plus, l'année prochaine, on attaque un gîte. [...] On s'est dit, ben tiens, ça fera un peu la retraite. Si on a des problèmes de santé, voilà, ça fera un petit revenu. Et après de toute façon, entre nous y a une solidarité, on est mariés, tout ce qui est là est à tous les deux, enfin bon, on n'a pas envie de payer, voilà, on n'a pas envie de payer la MSA.

rémunérée, et activité rémunérée est difficile à mettre en évidence, et il est courant de voir les chefs d'entreprise recourir aux différents membres de la famille pour des heures de travail, plus ou moins rémunérées sur le mode du « coup de main » ponctuel (voir entre autres Weber, 2008 ; ZarCA, 1993 ; Guichard, Poussou-Plesse, 2017). 
S : - C'est vrai que j'aurais pu être cotisant solidaire, ne serait-ce que conjoint d'exploitant.

F : - Faudrait peut-être qu'on se renseigne. [À moi] Je sais pas si vous connaissez...

S : - Voilà, ça fait un moment, chaque fois j'en parle et pff... je m'en occupe pas. »

Mariés et copropriétaires du terrain et de la maison qu'ils ont construite, Samia et Florent n'envisagent à aucun moment l'hypothèse d'une rupture conjugale. La stratégie économique qu'ils ont adoptée fait reposer la solidarité sur le couple (le mariage, le gîte pour assurer la retraite) plutôt que sur le corps social dans son ensemble. La non-déclaration n'est pas perçue par ces agriculteurs comme une question morale. Elle s'inscrit dans une rationalité économique délibérément en marge et déviante vis-à-vis de la norme dominante (le couple est contre la spéculation et refuse de faire reposer son assurance retraite sur un système spéculatif, des assurances-vie par exemple), mais aussi de l'État, qui n'incarne pas dans leurs schèmes de pensée un système véritablement solidaire. De ce point de vue, on peut voir les inégalités de genre en termes de statut professionnel comme l'une des manifestations possibles de l'ethos « alternatif » des agriculteur·rice·s « néo-ruraux·ales ». Dès lors, et alors qu'ils n'ont pas les ressources économiques suffisantes pour assurer une protection sociale à chacun, les couples d'agriculteur·rice·s renvoient dos à dos les structures économiques et les dispositifs publics, en même temps qu'ils valorisent les relations personnelles et la solidarité interindividuelle.

\section{Le poids de la méconnaissance des dispositifs et des risques}

Si ces agricultrices font reposer leur protection sociale sur leur couple, c'est aussi parce qu'elles méconnaissent tant les risques véritablement encourus que les manières de s'en prémunir. On observe parfois une relative ignorance des régimes statutaires accessibles aux conjointes et des droits qui y sont associés, comme le montrent les propos qui précèdent de Florent Verdier au sujet du statut de conjoint·e collaborateur-rice. Dans ces situations, le mariage et la copropriété des biens (maison et/ou terrains cultivés) semblent faire écran aux formes instituées et collectives de protection sociale ( « entre nous y a une solidarité, on est mariés, tout ce qui est là est à tous les deux »).

De plus, ces agriculteur·rice·s ne prennent pas la mesure des risques économiques induits par la rupture conjugale, et ignorent notamment que la contribution économique des femmes dans les exploitations agricoles, qu'elle soit productive ou seulement financière, est largement sous-reconnue par l'institution judiciaire dans les séparations conjugales d'agriculteurs (BESsiÈRE, GOLLAC, 2014). C'est en tout cas ce que suggère le cas d'Anne Ragon (46 ans) qui, constatant les effets du divorce de son beau-frère sur son ex-belle-sœur, a remis en cause son inexistence statutaire. Fille d'un couple d'éleveurs vendéens, Anne est la conjointe de Stéphane Muller, ce maraîcher d'origine allemande installé dans le village de Tournot depuis le début des années 1980 (cf. supra). Mais elle n'était pas là au moment de l'installation de Stéphane, qui était 
alors en couple avec une autre femme. Son arrivée à Tournot au début des années 1990 s'opère au terme d'un parcours de sortie du «monde agricole », fait de voyages à l'étranger (en Autriche et « un peu partout »), de vie salariée (dans une imprimerie) et urbaine (à Paris), de reprise d'études universitaires (Deug [diplôme d'études universitaires générales] de philosophie), et d'action collective contre le mal-logement. D'abord bénévole sur l'exploitation de Stéphane, Anne va rester pendant plus de quinze ans une travailleuse non déclarée sur l'exploitation de son conjoint : ce n'est qu'en 2008 que le couple décide de se marier ${ }^{31}$ et d'inscrire Anne comme conjointecollaboratrice à la MSA. En effet, en 2007, l'accident de travail subi par Stéphane le laisse inactif pendant plusieurs mois, ce qui incite Anne à repenser sa situation : elle comprend qu'il est important d'avoir une protection sociale en adéquation avec son statut de travailleuse à plein-temps. Elle prend également conscience, nous l'avons dit, de la précarité de son statut de travailleuse invisible à l'occasion du divorce de son beau-frère Alain d'avec sa compagne Nila. Tous deux vivent dans une maison mitoyenne et partagent avec Anne et Stéphane la propriété du terrain sur lequel tous les quatre sont installés (Anne et Stéphane font du marâichage biologique ; Alain et Nila y élèvent des brebis). En provoquant des conflits relatifs à la gestion du troupeau, ce divorce permet à Anne de se rendre compte de la nécessité de se protéger a minima contre l'éventualité d'un divorce :

« Avant, jusqu'à ce que je me marie, j'étais au black ici, tout en payant, en travaillant tout ça. Bon, c'est pareil, tu t'en fous tout un moment et puis un jour tu te dis, peut-être quand même, tu sais jamais ce qui peut se passer. En plus il commençait à y avoir les histoires là-haut, tu dis, bon, attends ouais, parce que du coup, Nila, elle a aussi ce statut de conjointe collaboratrice et du coup, elle a aucun pouvoir. Bon, c'est le gros bordel entre eux là, par rapport au troupeau machin, alors que tout s'est fait ensemble. C'est un peu tout ça qui nous a donné l'idée de régulariser au mieux l'histoire. Qu'il y ait un minimum de protection quand même. »

Le cas d'Anne Ragon révèle ainsi le caractère potentiellement provisoire du travail invisible des femmes enquêtées. Dès lors que s'opère la prise de conscience des risques encourus, et une fois les conditions économiques réunies pour honorer les cotisations sociales, la reconnaissance statutaire de ces agricultrices devient alors concevable. Samia Verdier et son conjoint Florent, de même que Suzanne Fèvre et son conjoint Jean-Paul, évoquent d'ailleurs en entretien avoir déjà songé à remédier à cette situation d'invisibilité statutaire (sans pour autant avoir concrétisé cette intention). Plus qu'un objet de conflit entre femmes et hommes et de résistance masculine à la reconnaissance du travail féminin (ZARCA, 1993), l'inégalité de genre mise en évidence ici apparait comme un impensé et une non-priorité, pour ces agriculteur-rice-s soucieux-ses en premier lieu de l'équilibre économique fragile de leur entreprise. Mais le danger est alors grand que cette démarche survienne trop tard pour qu'elle ait un intérêt

31. Sous le régime de la communauté réduite aux acquêts, ce qui l'a rendue propriétaire d'un huitième de l'exploitation (suite au rachat par Stéphane de la part de l'exploitation appartenant à son ex-femme). 
économique et social réel, comme le montre avec force le cas de Brigitte Serres, et que se prolonge ainsi la situation de dépendance dans laquelle sont placées ces femmes.

En agriculture, au cours des dernières décennies, les femmes ont gagné en reconnaissance et ont des trajectoires professionnelles de plus en plus indépendantes de celles de leur conjoint. Mais ce phénomène se déploie de façon différenciée et certains espaces sociaux se caractérisent par la permanence du travail en couple et de l'invisibilité du travail féminin. Dans cette enquête menée auprès des maraîcher-ère-s biologiques des Alpes-Maritimes, il apparaît que la quasi-totalité des agriculteur-rice-s «néoruraux ales » travaillent en couple, et souvent sans statut professionnel. En interrogeant les logiques sociales qui conduisent ces femmes à devenir agricultrices aux côtés de leur conjoint et à travailler dans l'ombre de celui-ci, on aura montré premièrement que si l'installation «néo-rurale » constitue aujourd'hui le point d'aboutissement d'une rupture ou d'une réorientation professionnelle, la concrétisation en couple d'un nouveau projet professionnel obéit principalement à des logiques familiales. Et si la prise en charge des enfants et la construction d'un avenir social, professionnel et familial, fonctionnent bien comme facteurs de convergence des aspirations professionnelles des couples d'agriculteur-rice-s « néo-ruraux-ales », ils semblent fonctionner surtout dans un sens : celui qui incite les femmes salariées à quitter leur emploi et à s'investir dans le projet agricole de leur conjoint. Deuxièmement, l'invisibilité statutaire de ces agricultrices est d'abord le produit de contraintes économiques : parce qu'ils se situent le plus souvent dans une économie de la survie, ces agriculteur.rice-s sont contraintee.s de s'accommoder de configurations statutaires inégalitaires en termes de genre. Mais ces rapports inégaux s'expliquent aussi par la méconnaissance relative des risques encourus et des manières de s'en prémunir, et par une certaine défiance à l'égard des formes instituées de protection sociale qui les conduit à privilégier la solidarité de couple et à élaborer des stratégies individuelles compensatoires. Dans ces situations, le recours aux affects (l'engagement conjugal), à l'entraide et à la réciprocité, ou encore à l'argument du caractère non marchand et « alternatif » de leurs pratiques économiques, constitue un arrangement pratique permettant de neutraliser les tensions créées. 


\section{BibliograPhIE}

BAJARD F. (2014), Sociologie des céramistes d'art en France. L'invention d'un groupe socioprofessionnel : pratiques et manières d'être, thèse de doctorat en sciences sociales, Université de Lausanne.

Bajard F., Perrenoud M. (2013), « “Ça n'a pas de prix”. Diversité des modes de rétribution du travail des artisans d'art », Sociétés contemporaines, n 91, pp. 93-116.

BARTHEZ A. (1982), Famille, travail et agriculture, Paris, Economica.

BertauX-Wiame I. (1982), «L'installation dans la boulangerie artisanale », Sociologie du travail, vol. 24, $\mathrm{n}^{\circ} 1$, pp. 8-23.

BESSIÈRE C. (2010), De génération en génération. Arrangements de famille dans les entreprises viticoles de Cognac, Paris, Raisons d'agir.

Bessière C., Bruneau I., LAFERTÉ G. (2014), « Introduction. Les agriculteurs dans la France contemporaine », Sociétés contemporaines, ${ }^{\circ}$ 96, pp. 5-26.

Bessière C., Giraud C., Renahy N. (2008), « Introduction - Famille, travail, école et agriculture », Revue d'études en agriculture et environnement, vol. 88, nº 1, p. 5-19.

BESSIÈRE C., GollaC S. (2014), « Des exploitations agricoles au travers de l'épreuve du divorce. Rapports sociaux de classe et de sexe dans l'agriculture », Sociétés contemporaines, $\mathrm{n}^{\circ}$ 96, pp. 77-108.

Bidet A. (2011), L'Engagement dans le travail. Qu'est-ce que le vrai boulot?, Paris, Presses universitaires de France.

BisAult L. (2009), «Agricultrice : un métier qui s'impose à tout petits pas », Agreste primeur, $\mathrm{n}^{\circ} 223$.

Bruneau I. (2006), La Confédération paysanne. S'engager à « juste » distance, thèse de doctorat en science politique, Université Paris X.

CAniou J., Lagrave R.-M. (1988), « Un statut mis à l'index », in Lagrave R.-M. (dir.), Celles de la terre. Agricultrice : l'invention politique d'un métier, Paris, Éditions de l'École des hautes études en sciences sociales, pp. 111-150.

CARDON P. (2004), Des femmes et des fermes. Genres, parcours biographiques et transmission familiale. Une sociologie comparative Andalousie - Franche-Comté, Paris, L'Harmattan.

CASTEL R. (1995), Les Métamorphoses de la question sociale. Une chronique du salariat, Paris, Fayard.

Denave S. (2015), Reconstruire sa vie professionnelle. Sociologie des bifurcations biographiques, Paris, Presses universitaires de France.

Dufour A., GiRAud C. (2012), « Le travail dans les exploitations d'élevage bovin laitier est-il toujours conjugal ? », INRA productions animales, vol. 25, $\mathrm{n}^{\circ} 2$, pp. 169-180.

ESCUDIER J.-L. (2017), « L'emploi et la protection sociale dans l'agriculture au filtre des rapports de genre. Les salariées agricoles aux XIX ${ }^{\mathrm{e}}$ et XX ${ }^{\mathrm{e}}$ siècles », Travail et Emploi, $\mathrm{n}^{\circ}$ 149, pp. 73-97. 
Gillet M., JacQues-Jouvenot D. (2004), « La dépendance dans l'indépendance », Cahiers du genre, $\mathrm{n}^{\mathrm{o}} 37$, pp. 171-190.

GiRAud C., RÉmy J. (2008), «Le choix des conjoints en agriculture », Revue d'études en agriculture et environnement, vol. 88, $\mathrm{n}^{\circ} 1$, pp. 21-46.

Gojard S. (2010), Le Métier de mère, Paris, La Dispute.

GollaC S. (2003), « Maisonnée et cause commune : une prise en charge familiale », in Weber F., Gojard S., Gramain A. (dir.), Charges de famille. Dépendance et parenté dans la France contemporaine, Paris, La Découverte, pp. 274-311.

Gollac S. (2013), « Propriété immobilière et autochtonie. Les trajectoires immobilières, sociales et politiques d'un maçon breton et de ses descendant·e·s », Politix, n ${ }^{\circ}$ 101, pp. 133-159.

Guichard M., Poussou-Plesse M. (2017), « L'artisan, son comptable et sa retraite. Étude d'un profil d'indépendants établis », Travail et Emploi, $\mathrm{n}^{\circ}$ 149, pp. 43-72.

LÉGer D., Hervieu-LÉGer B. (1979), Le Retour à la nature. "Au fond de la forêt... l'État », Paris, Seuil.

Hirschman A. ([1970], 1995), Défection et prise de parole. Théorie et applications, Paris, Fayard.

LAGRAVE R.-M. (dir.) (1988), Celles de la terre. Agricultrice : l'invention politique d'un métier, Paris, Éditions de l'École des hautes études en sciences sociales.

LAisney C., Lerbourg J. (2012), « Les femmes dans le monde agricole », Analyse, $\mathrm{n}^{\circ} 38$, Montreuil, Centre d'études et de prospective.

LANDOUR J. (2012), « Quitter l'entreprise pour renouer avec le travail ? Les parcours de désengagement de cadres d'un institut d'études », Sociologie du travail, vol. 54, n 4, pp. 511-532.

LANDOUR J. (2017), « Les Mompreneurs. Entre entreprise économique, identitaire et parentale », Travail et Emploi, $\mathrm{n}^{\circ}$ 150, pp. 79-100.

PaGis J. (2015), Mai 68, un pavé dans leur histoire. Événements et socialisation politique, Paris, Presses de Sciences Po.

Perez C. (2014), « La déstabilisation des stables : restructuration financière et travail insoutenable », Travail et Emploi, $\mathrm{n}^{\circ} 138$, pp. 37-52.

Pruvost G. (2013), « L'alternative écologique. Vivre et travailler autrement », Terrain, $\mathrm{n}^{\circ}$ 60, pp. 36-55.

RatTin S. (2006), «En agriculture, la parité n'est pas de mise », Agreste primeur, $\mathrm{n}^{\circ} 175$.

RETIÈRE J.-N. (2003), « Autour de l'autochtonie. Réflexions sur la notion de capital social populaire », Politix, n ${ }^{\circ}$ 63, pp. 121-143.

SАMAK M. (2014), Un engagement par le travail ? Enquête sur les maraîchers biologiques des Alpes-Maritimes, thèse de doctorat en sociologie, École des hautes études en sciences sociales.

SAMAK M. (2016), « La politisation variable des alternatives agricoles », Savoir/Agir, $\mathrm{n}^{\circ} 38$, pp. 29-35. 
SAMAK M. (2016), «Pratiques d'emploi et figures du patron en agriculture biologique. Contribution à une sociologie du travail indépendant », Sociologie du travail, vol. 58, $\mathrm{n}^{\circ} 4$, pp. 412-434.

WeBER F. (2000), «Transactions marchandes, échanges rituels, relations personnelles. Une ethnographie économique après le Grand Partage », Genèses, n ${ }^{\circ}$ 41, pp. 85-107.

WEBER F. (2008), Le Travail au noir, une fraude parfois vitale? Une conférence-débat de l'association Emmaüs, 30 janvier 2008, Paris, Éditions Rue d'Ulm/Presses de l'École normale supérieure.

Weber F. ([1989], 2009), Le Travail à-côté. Une ethnographie des perceptions, Paris, Éditions de l'École des hautes études en sciences sociales.

ZARCA B. (1993), «Indépendance professionnelle, relations entre les sexes et mobilisations collectives », Sociétés contemporaines, $\mathrm{n}^{\circ}$ 16, pp. 77-109. 


\section{ANNEXE - LISTE RÉCAPITULATIVE DES FAMILLES D'EXPLOITANT•E•S ENQUÊTÉES}

\section{Agriculteur·rice·s « néo-ruraux·ales »}

CASTAIN

* Exploitation : 1,8 ha de maraîchage

† Castain Laure : cheffe d'exploitation, 46 ans*, père cadre du privé, mère au foyer

$\infty$ Situation conjugale : célibataire, séparée

\section{FAURE}

* Exploitation : 1 ha de maraîchage

$q$ Faure Julia : cheffe d'exploitation, 31 ans, père cadre du privé, mère au foyer

$\widehat{\gamma}$ Faure Laurent : sans statut, 32 ans, père cadre du privé, mère secrétaire de direction

\section{FÈVRE}

- Exploitation : 1 ha de maraîchage

T Fèvre Jean-Paul : chef d'exploitation, 55 ans, parents ouvriers cheminots

F Fèvre Suzanne : sans statut, 52 ans, parents commerçants

\section{MÉRIEUX}

*xploitation : 1,4 ha de marâichage

đấrieux Michel : chef d'exploitation, 62 ans, père technicien cheminot, mère institutrice

$\infty$ Situation conjugale : marié, conjointe travaillant à l'extérieur de l'exploitation

\section{MONGIN-ROSTAN}

* Exploitation : 1 ha de marâichage

đostan Gautier : chef d'exploitation, 38 ans, parents agents des impôts

+ Mongin Chloé : salariée à temps partiel à l'extérieur de l'exploitation (provisoire), 33 ans, parents enseignants

\section{MULLER-RAGON}

* Exploitation : 1,5 ha de maraîchage

3. Muller Stéphane : chef d'exploitation, 51 ans, père pasteur, mère au foyer

q Ragon Anne : conjointe collaboratrice depuis 2008, sans statut de 1992 à 2008, 46 ans, parents agriculteurs

\section{MARINI-SERRES}

* Exploitation : 2,2 ha de maraîchage

đT Marini Gérard : chef d'exploitation, 60 ans, père sous-officier de l'armée, mère au foyer

o Serres Brigitte : sans statut, 62 ans, parents commerçants

\section{REVEL}

- Exploitation : 0,5 ha de maraîchage

q Revel Monique : cheffe d'exploitation, 55 ans, père dessinateur industriel, mère au foyer

$\delta^{T}$ Revel Jean-Pierre : retraité, 58 ans, père maçon, mère au foyer

\section{TISSOT-MARTIN}

* Exploitation : 1,2 ha de maraîchage

ô Tissot Christophe : chef d'exploitation, 36 ans, père technicien dans l'industrie, mère au foyer

+ Martin Carole : sans statut, 30 ans, profession des parents inconnue

\section{VERDIER}

\&xploitation : 1 ha en maraîchage

T) Verdier Florent : chef d'exploitation, 40 ans, père comptable, mère secrétaire

q Verdier Samia : sans statut, 38 ans, père ouvrier, mère au foyer

\footnotetext{
* Par convention, l'âge des enquêtée··s a été calculé par rapport à l'année 2010.
} 


\section{Agriculteur.rice.s repreneur-se-s d'exploitation}

\section{BRUNO}

Exploitation : 2 ha de maraîchage

B Bruno Patrick : coexploitant, 51 ans, père employé comptable, mère au foyer

$\Varangle$ Bruno Christine : coexploitante, 46 ans, parents agriculteurs

\section{CARATTI}

- Exploitation : 8 ha de maraîchage

$\widehat{~ C a r a t t i ~ P a u l ~: ~ c h e f ~ d ' e x p l o i t a t i o n, ~} 74$ ans, père contremaître dans l'industrie, mère au foyer

+ Caratti Mireille : conjointe collaboratrice, 68 ans, parents agriculteurs

\section{CARDAN}

Exploitation : 1,5 ha de maraîchage

$\delta$ Cardan Philippe : chef d'exploitation, 50 ans, parents agriculteurs

+ Cardan Sanya : conjointe collaboratrice, 43 ans, parents agriculteurs

\section{GARRIDO}

Exploitation : 2,5 ha de maraîchage

7. Garrido Antoine : chef d'exploitation, 56 ans, parents agriculteurs

† Garrido Élise : conjointe collaboratrice, 60 ans, père serveur-chef de rang, mère au foyer

\section{LAMY}

Exploitation : 1 ha de maraîchage

¿ Lamy Victor : chef d'exploitation, 56 ans, parents agriculteurs

$\infty$ Situation conjugale : marié, conjointe travaillant à l'extérieur de l'exploitation

\section{MASSIERA}

* Exploitation : 1,2 ha de maraîchage

+ Massiera Marie : cheffe d'exploitation, 51 ans, père ouvrier, mère employée de mairie

$\infty$ Situation conjugale : célibataire, divorcée

\section{MURET}

* Exploitation : 0,8 ha de maraîchage

\. Muret Pierre : chef d'exploitation, 57 ans, parents agriculteurs

$\infty$ Situation conjugale : célibataire, divorcé

\section{OLIVETTI}

* Exploitation : 4,7 ha de maraîchage

đolivetti Éric : chef d'exploitation, 52 ans, père cadre du privé, mère au foyer

O Olivetti Florence : conjointe collaboratrice, 50 ans, parents agriculteurs

\section{PRÉVOT}

* Exploitation : 0,8 ha de maraîchage

+ Prévot Janine : cheffe d'exploitation, 57 ans, père conducteur de travaux, mère institutrice

đ Prévot André : chef d'entreprise (pour une pépinière connexe à l'exploitation agricole), 57 ans, parents agriculteurs

PUJOL

* Exploitation : 4,5 ha de maraîchage

o Pujol Roland : chef d'exploitation, 52 ans, parents agriculteurs

$\infty$ Situation conjugale : concubinage, conjointe travaillant à l'extérieur de l'exploitation

\section{ROSSO}

- Exploitation : 3 ha de maraîchage

$\delta$ Rosso Bernard : chef d'exploitation, 62 ans, parents agriculteurs

$\infty$ Situation conjugale : marié, conjointe travaillant à l'extérieur de l'exploitation

\section{SOREL}

* Exploitation : 0,4 ha de maraîchage

† Sorel Colette : cheffe d'exploitation, 46 ans, parents maraîchers

$\infty$ Situation conjugale : mariée, conjoint travaillant à l'extérieur de l'exploitation (mais projetant de s'investir sur l'exploitation à temps partiel) 